\title{
Microphthalmia-Associated Transcription Factor in Senescence and Age-Related Diseases
}

\author{
Jian Zhang ${ }^{\mathrm{a}}$ Yi Mou $^{\mathrm{b}}$ Hui Gong ${ }^{\mathrm{a}}$ Honghan Chen $^{\mathrm{a}}$ Hengyi Xiao $^{\mathrm{a}}$ \\ aLab for Aging Research, National Clinical Research Center for Geriatrics, State Key Laboratory of Biotherapy, \\ West China Hospital of Sichuan University, Chengdu, China; ${ }^{b}$ Geroscience and Chronic Disease Department, \\ The 8th Municipal Hospital for the People, Chengdu, China
}

\section{Keywords}

Microphthalmia-associated transcription factor ·

Melanocyte $\cdot$ Senescence $\cdot$ Degenerative diseases

\begin{abstract}
Although microphthalmia-associated transcription factor (MITF) has been known for decades as a key regulator for melanocytic differentiation, recent studies expanded its other roles in multiple biological processes. Among these newfound roles, the relationship between MITF and aging is attractive; however, the underlying mechanism remains elusive. Here, we review the documented cues that highlight the implication of MITF in the aging process and particularly discuss the possible mechanisms underlying the participation of MITF in cellular senescence. First, it summarizes the association of MITF with melanocytic senescence, including the roles of MITF in cell cycle regulation, DNA damage repair, oxidative stress response, and the generation of senescenceassociated secretory phenotype. Then, it collects the information involving MITF-related senescent changes in nonmelanocytes, such as retinal pigment epithelium cells, osteoclasts, and cardiomyocytes. This review may deepen the understanding of MITF function and be helpful to develop new strategies for improving geriatric health.
\end{abstract}

(c) 2021 S. Karger AG, Basel

\section{Introduction}

Microphthalmia-associated transcription factor (MITF) was first discovered by Hertwig [1] who stated the special phenotypes found in the descendant of an irradiated male mouse, which include small eyes, white hair, hearing deficiency, and the link between a locus mutation that was later named as $\mathrm{mi}$ [2]. Subsequent studies found that mice with mutations at the mi locus also have problems such as abnormal bone resorption and early onset of deafness $[3,4]$. Considering the role of MITF in the survival and differentiation of melanocytes and the dominant expression in pre-melanocytes and melanocytes, MITF was further referred to as melanocyte-inducing transcription factor [5]. However, recent studies showed that MITF is also expressed in retinal pigment epithelial (RPE) cells [6], osteoclasts [7], and cardiomyocytes [8]. The dysfunction of these cells is closely related with retinal degradation, bone loss, and cardiac weakness, the typical signs of physical decrepitude.

Aging is a universal feature of all living organisms and is characterized by irreversible progressive functional loss accompanied by anatomical or histological degeneration, due to interactions between genetic and environmental factors. Aging at the cellular level, generally referred to senescence, is a nondividing status of stable cell cycle ar-

\footnotetext{
Karger"
}

Correspondence to:

Hengyi Xiao, hengyix@scu.edu.cn 
rest [9]. Senescence is categorized as replicative senescence and stress-induced premature senescence (SIPS). The most fundamental cellular change in replicative senescence is the shortening of telomeres, while the cellular changes in SIPS are more complex, depending on the stresses imposed, such as over-activated oncogenes, ionizing radiation, and oxidative stress [10]. Importantly, cellular senescence is closely associated with age-related pathogenesis and has become an essential cause of tissue dysfunction and aging-related diseases [11]. Regrettably, however, many questions underlying the mechanisms of senescence remain unanswered, largely because we still lack knowledge about the functional molecules involved in or counteracting this process.

Importantly, MITF plays a vital role in melanin synthesis, and some mice with MITF mutations display aging-related phenotypes, such as pigment loss and osteoporosis $[3,4]$. These findings suggest that MITF may have superior biochemical properties against environmental risk factors, particularly oxidative stress and ultraviolet radiation (UVR), and therefore would be essential for deferring senility. Although many knowledge gaps need to be filled, the available information provides a rational for evaluating the significance of MITF modulation for aging and senescence intervention. Although whether and how MITF regulates melanin production from melanocytes in response to various scenarios are still largely unknown, exploring the biological function of MITF in aging and age-related diseases could be an interesting research topic.

\section{MITF and Its Classic Function}

\section{MITF and Its Family}

In 1993, mi was identified by Hodgkinson et al. [12] in mice as a novel gene encoding a basic helix-loop-helixleucine zipper (bHLH-Zip) protein. It was not until 1994 that Tachibana et al. [13] cloned MITF as the human homolog of mouse mi and assigned it to chromosome 3p14.1-p12.3. Most meanwhile, Tassabehji et al. [14] reported that the mutation of human MITF leads to Waardenburg syndrome type 2 . Now, it is known that MITF belongs to the micropthalmia family (MiT family, coding bHLH-Zip DNA-binding structure) together with other 3 transcriptional factors, which are TFEB, TFE3, and TFEC [13]. MiT family has conserved sequences at the nucleic acid and activates target gene expression by directly binding DNA as homo- or heterodimers [15]. Similar to other members of the larger family of HLH leucine-zipper transcription factors, the DNA element

MITF in Senescence and Age-Related Diseases bound by MiT proteins features commonly as a palindromic DNA sequence (CACGTG) located in the proximal promoter of target genes (E-box) [16].

The function and regulatory mechanisms of MiT proteins have some overlap. However, the specific function of each family member is somewhat unique, owing to its expression pattern and different structure. TFEB and TFE3 are involved in the transcriptional network of lysosome biogenesis, cellular energy homeostasis, and autophagy, where TFEB is a master regulator $[17,18]$. Because of the lack of the acidic domain, the power of transcription activation of TFEC is weaker than that of TFE3 [19]. However, TFEC plays an important role in the niche to expand hematopoietic progenitors during zebrafish embryogenesis [20]. Since the discovery of its association with fur color and eye development in mice and rats, MITF has been particularly looked at for its role in the regulation of melanocyte survival and differentiation, melanosome biogenesis, and eye development [21]. Interestingly, studies have found that TFEB and its ortholog HLH-30 in Caenorhabditis elegans are essential for dietinduced steatosis and the extended life span [22, 23]. It indicates that other members of the MiT family, such as MITF, may own some kind of connection with age or agerelated diseases.

\section{MITF and Melanocyte Development}

MITF has 9 isoforms (including MITF-A, MITF-J, MITF-C, MITF-MC, MITF-E, MITF-H, MITF-D, MITF$\mathrm{B}$, and MITF-M) with 1 bHLH-Zip DNA-binding domain and 2 trans-activation domains [24]. Being complicated, different MITF isoforms regulate unique and overlapping gene sets, with cell- and tissue-specific distribution. The shortest isoform, MITF-M, is exclusively expressed in melanocyte and melanoma cells under the control of the unique melanocyte-restricted intron promoter [25].

Many studies have revealed insights into central roles of MITF in melanocytes, including the aspects of differentiation and proliferation. In mice, $>20$ different MITF mutations are associated with the lack of neuronal crestderived melanocytes. MITF first promotes the transformation of progenitor cells into melanocytes and then promotes the survival of melanocytes by affecting the expression of Kit [26]. Moreover, its ortholog in zebrafish is required for the development of melanocytes [27]. Further studies showed that MITF actually mediates tyrosinase expression and melanocyte differentiation induced by the cAMP pathway [28], and overexpression of MITF can induce embryonic stem cells to differentiate into me- 
lanocytes [29]. In addition, MITF can directly regulate the expression of other proteins involved in melanocyte survival (Bcl2, BIRC7, MET, HIF1A, and APEX1) and growth (CDK2, DIAPH1, TBX2, p16INK4A, and p21Cip1) [30]. This prominent role follows that MITF is a key regulator of melanocyte development.

\section{Implications of MITF in Melanocyte Senescence}

In a clinical study involving 63 patients with intermediate-thickness $(1.0-4.0 \mathrm{~mm})$ melanoma, the average overall survival time of MITF-positive melanoma patients was significantly higher than that of MITF-negative melanoma patients $(187.90 \pm 13.41$ vs. $80.89 \pm 17.98$ months, respectively, $p=0.0086$ ). After lymph node dissection, patients with MITF expression $>50 \%$ had significantly less lymph node metastasis than those with MITF expression $\leq 50 \%$ [31]. This indicates that MITF suppresses tumor metastasis and prolongs patient survival in intermediate-thickness melanoma. Moreover, it demonstrates that the expression level of MITF is associated with the life span of melanoma patients. Ko and Kim [32] reported that the expression of MITF proteins was downregulated in senescent and $\mathrm{H}_{2} \mathrm{O}_{2}$-treated melanocytes. This suggests that $\mathrm{H}_{2} \mathrm{O}_{2}$-induced senescence is negatively associated with the expression level of MITF in melanocytes. Combining this information and the earlier results showing the role of MITF in the aging process, we think it is worthy to explore more about the implication of MITF in cellular and biological aging. Based on the current research actuality, we first try to summarize the biological connection of MITF with cellular senescence, particularly in melanocytic senescence. The information is grouped into several aspects, sorting out by the major features of senescence, such as cell cycle arrest, DNA damage, oxidative stress response, and senescence-associated secretory phenotype (SASP) production.

\section{Role of MITF in Cell Cycle}

Unlike dormant cells, senescent cells are stable, arrested in G1 phase, and cannot proliferate effectively. The barrier to unlimited growth existing in senescent cells is mainly driven by the activation of at least 2 important signaling pathways: the $\mathrm{p} 53 / \mathrm{p} 21$ pathway and the $\mathrm{Rb} /$ p16INK4A pathway [33]. The association of MITF with the cell cycle is complex, likely depending on its activity and expression.

The MITF is commonly thought to be a melanoma oncogene [34]. For example, a mutation of MITFE318K se- verely impairs the SUMOylation of MITF protein and elevates the transcriptional activity of MITF and the risk of melanoma development compared to those of the wildtype MITF [35]. Importantly, MITFE318K carrying melanocytes have stronger proliferative activity but lower expression of p16 and p21; additionally, this kind of mutation of MITF delays the senescence process of BRAFV600E-induced melanocytes [36]. Furthermore, MITF has been shown to control the transcription of TBX2 [37], which in turn prevents senescence by inhibiting p21 and is involved in the control of melanocyte growth and invasion [38]. Giuliano et al. [39] found that sustained inhibition of MITF expression can trigger the senescence of melanoma cells by inducing an increase in p16INK4A and p53 and a decrease in CDK2. Despite the above results, contradictory findings were also reported, showing that high level of MITF increases p16INk4A expression and triggers cell cycle arrest by directly binding to the INK4A promoter [40]. MITF also can induce G1 arrest by upregulating the expression of $\mathrm{p} 21$ and consequent hypophosphorylation of Rb1 [41] or P53 activation [42]. In addition, MITF can directly upregulate cell cyclin-related genes CCNB1 and CCND1 and the mitotic gene PLK1 [43].

Given these contradictory observations, the significance of MITF in cell proliferation seems complicated and time- or context-dependent. More concretely, it probably depends on the cellular metabolism, the functional compensation of other proteins, the variation in MITF expression, and so on. Anyway, it is clear that MITF plays a role in the cell cycle regulation at least in melanocytes, and most data show it is pro-proliferative but antiG1 arrest.

\section{Role of MITF in DNA Damage Repair}

Persistent DNA damage can retard transcription and replication, thus hindering cell function and trigging senescence [44]. Melanin protects cells particularly from UVR-induced DNA damage. If not repaired, UV lightinduced DNA lesions can lead to various skin abnormalities and photoaging. After DNA damage, PKA-mediated cascade quickly induces the transcription of MITF and then melanin production, which protects photoaging [28].

Several studies have shown that MITF is involved in DNA replication, DNA damage repair, and chromosome integrity. For instance, MITF depletion-triggered senescence in melanoma cells is accompanied with mitotic defects, DNA damages, and the activated ATM/ $\gamma \mathrm{H} 2 \mathrm{AX} / 53 \mathrm{BP} 1 / \mathrm{CHK} 2 / \mathrm{p} 53$ cascade [39]. Likely, the 
knockdown of MITF prompts the senescence of melanoma cells and downregulates the expression of 15 genes, working for DNA replication, recombination, and repair, such as LIG1, TERT, EME1, BRCA1, and FANCA, and the expression of 39 genes, working for centromere organization and mitosis, such as HAUS8, SPC24, CCNB1, CCND1, and PLK1 [43]. A similar expression profile revealed that MITF activity is associated with the expression of a series of genes involved in DNA damage response and repair based on immunoblot assays [45]. Conversely, in zebrafish, the loss of MITF leads to an obvious decrease in pigmentation gene expression but an increase in DNA repair gene expression, including ATR, $\mathrm{XPA}, \mathrm{ERCC1}$, LIH4, and TDG [46]. Interesting, it is known that MITF also acts for the rapid recovery of nucleotide excision repair through transactivating GTF2H1 (the core element of TFIIH) and CDK7 (TFIIH kinase) [47]. In summary, MITF is an active transcription factor involved in the regulation of DNA damage and repair, which makes its interaction with DNA damage-related senescence conceivable.

\section{Role of MITF in Oxidative Stress Response}

Melanin production in melanocytes is the first line of defense in protecting the skin from photoaging caused by solar radiation, while the latter is a typical kind of oxidative stimuli, such as UVR and $\mathrm{H}_{2} \mathrm{O}_{2}$, quickly evoking oxidative stress and relevant response in skin cells [48]. Therefore, the control of oxidative stress is of importance in preventing skin aging and melanocyte proliferation.

In fact, MITF affects the expression of key components working for oxidative stress defense. First explored by Jiménez-Cervantes et al. [49], MITF expression is correlated with $\mathrm{H}_{2} \mathrm{O}_{2}$-induced oxidative stress and melanin production is inhibited in melanocytes with a low expression of MITF in $\mathrm{H}_{2} \mathrm{O}_{2}$-treated mouse and human melanoma cells. Liu et al. [50] reported later that decreasing MITF can weaken the induction of reactive oxygen species (ROS) by directly reducing the accumulation of redox sensor APE-1. MITF also strongly stimulates the transcriptional activity of hypoxia-inducible factor 1-alpha by binding the promoter of HIF1 $\alpha$ gene [51]. In addition, MITF has been verified to be a direct transcription activator of human PGC1 $\alpha$ gene, actually enabling melanoma cells to survive under oxidative stress, by improving mitochondrial energy metabolism and ROS detoxification capabilities [52]. Moreover, overexpression of MITF can induce the expression of oxidative phosphorylation genes working for mitochondrial homeostasis and significantly increase mitochondrial metabolism. Given

MITF in Senescence and Age-Related Diseases that mitochondria are the main source of ROS and its implication in mitochondria quality control, the contribution of MITF for photoaging caused by oxidative stress has received increasing attention in recent years. Despite PGC1 $\alpha$, MITF also controls the expression of some crucial components important for mitochondrial biogenesis [53]. Some researchers used various antioxidants and detoxification enzymes, such as melanocortin-1 receptor agonists pentapeptide [54], berberine [55], and coenzyme Q10 [56], to increase MITF expression together with the active Nrf2 pathway in order to enhance skin pigmentation and antioxidant defenses for anti-photoaging. Interestingly, stress response also impacts the transcriptional activity of MITF, such as the upregulated expression of ATF4, a crucial transcription factor in stress response, could activate the transcription of MITF by phosphorylation of eIF $2 \alpha$ and suppress the senescence of melanoma cells [57]. It demonstrates that MITF and its upstream/ downstream genes play a role in the oxidative stress and mitochondrial free radical theory of aging.

\section{Role of MITF in SASP Production}

In the last decade, increasing evidence ensured that senescent cells can secrete hundreds of factors, including pro-inflammatory cytokines, chemokines, growth factors, and proteases. This kind of secretion is called wildly as SASP [58]. The exact composition of SASP somewhat varies depending on cell status and cell context, as well as aging promoting stimuli. However, the key factors of SASP and its regulatory mechanisms seem to be conservative in senescent cells and tissues [59].

The association of MITF with SASP has been noticed, likely being mutual. On the one hand, compared to MITFpositive melanoma cells, MITF-negative melanoma cells produced larger amounts of IL- $1 \alpha$ and IL-1 $\beta$ [60]. Indeed, MITF inhibited premature senescence of melanoma cells induced by $\mathrm{H}_{2} \mathrm{O}_{2}$ and temozolomide and also suppressed chemokine CCL2 production. Consistently, the results from immunofluorescence assays, electrophoretic mobility shift assays, and luciferase reporter assays revealed that the depletion of MITF increased the transcriptional activity of NF- $\kappa B$ in melanoma cells, directly promoting the expression of various pro-inflammatory factors, the major components of SASP [61]. Moreover, silencing MITF in senescent melanoma cells exhibited an increase of the secretome, including SNAIL1, TWIST1, fibronectin1, and $\mathrm{N}$-cadherin (CDH2). On the other hand, however, IL-6 and STAT3 can induce a sharp decrease in the expression of MITF [62]. Although not absolutely, these observations clearly suggest the implication of MITF in 
SASP production, and the existence of a negative feedback loop between MITF and SASP production that leads gradually to reduced senescent phenotype gradually and increased the proliferative phenotype in cells [63]. It also indicates that the association between MITF and SASP production either regulates or is regulated by pro-inflammatory mechanism.

\section{Implications of MITF in Age-Related Diseases}

Actually, the biological roles of MITF are far beyond the matters of melanocytes. Accumulated evidence uncovers that MITF is also expressed and functions in cells or tissues other than melanocytes; the topic about the implications of MITF beyond melanocytes attracted increasing attention recently. In the following, we will summarize this set of information, with focus on the impacts of MITF in geriatric diseases, particularly retinal degeneration, osteoporosis, and cardiovascular diseases, mostly based on its existence and function in RPE cells, osteoclasts, and cardiomyocytes. We hope that these pieces of information are significant for the re-evaluation of MITF in aging process and can provide new insights on agingrelated diseases.

\section{Retinal Degeneration}

Retinal degeneration, manifested generally as age-related macular degeneration or retinitis pigmentosa, is not a rare condition, leading to irreversible blindness in the aged population. Therefore, exploring the underlying mechanisms of retinal degeneration is a prerequisite for preventing the progression of these diseases.

Structural abnormalities and dysfunction of RPE cells are considered to be the primary causes of retinal degeneration [64]. In this aspect, the role of MITF in the development and function of RPE cells has been expounded. First, MITF-A is highly expressed in RPE cells, and it activates the transcription of the genes encoding tyrosinase and tyrosinase-related protein 1 , which are the important factors of pigmentation [65]. Second, MITF regulates human RPE cell proliferation by indirectly upregulating the DAPL1 expression, an age-related macular degenerationsensitive protein [66]. In the third, MITF mutations resulted in RPE cell defects in mice, which led to the abnormal integrity and function of photoreceptors and even visual disability [67]. Follow-up studies indicate further that MITF mutations can cause abnormal expression of MITF-targeted genes that in turn lead to retinal degeneration [68]. Finally, it has been clarified that the lack of
MITF impedes various processes necessary for retinal function, including the ability of RPE cells to pigment formation, the visual cycle interruptions, and the eye functioning properly [69].

In fact, MITF acts as an antioxidant transcription factor to regulate mitochondrial biogenesis and redox signaling in RPE cells. For example, the level of ROS was significantly higher in MITF-/- mice than wild-type mice. MITF expression influences mitochondrial biogenesis of RPE cells by directly regulating PGC1 $\alpha$ and mitochondrial antioxidant enzymes to protect RPE cells from ROS-induced cellular damage [70], and MITF upregulates the Nrf2 pathway and then elevated the enzymatic antioxidants in RPE cells [71]. As excessive production of ROS in the retina can cause the degeneration and senescence of RPE cells [72], the antioxidative role of MITF should be a potential target for retinal protection. Coincidently, various antioxidants, either enzymatical or nonenzymatical, have been shown to prevent retinal degeneration in experimental animals and patients.

\section{Osteoporosis}

Osteoporosis is another retrogression that commonly happens in the elderly, being the fundamental cause of bone fracture and deformation of the spinal column. The implication of MITF in osteoporosis has been confirmed by multiple studies. The major cognitions include that MITF upregulates enhanced osteoclast formation or function and participates in the late stages of osteoclastogenesis and osteoclast terminal differentiation $[73,74]$. It has been found that 2 major isoforms of MITF, MITF-A and MITF$\mathrm{E}$, are expressed in osteoclasts, but MITF-E plays a more prominent role in osteoclastogenesis than MITF-A in mice and canines because it leads to the upregulation directly from the nuclear factor NF- $\mathrm{BB}$ ligand (RANK), a key regulator of osteoclast differentiation and formation [75]. It is worthy to mention here that the interplay between NF- $\kappa B$ and MITF seems reciprocal as MITF can increase the activity of RANK promoter by cis-element binding [76], and MITF-E acts as a distal transcription factor of the RANKL pathway and activates the transcription of NFATc1, the master regulator of osteoclastogenesis [77]. MITF has been reported to play an important role in the survival of osteoclast precursors, which is indispensable for functional osteoclast development [78]. One of the evidences revealed that the osteoclast precursor cells of MITF mutants displayed fusion defect, and mice showed the classic signs of osteoporosis, including short stature and increased radiopacity $[79,80]$. Anyway, normal expression of MITF is essential for osteoclast development and function. 
Fig. 1. Possible mechanism of MITF regulates the senescence process in melanocytes. When melanocytes suffer from external stimuli or internal stresses, the senescence program of melanocytes is triggered. Transcription factor MITF may involve in the senescence program of melanocytes by the following ways: (1) MITF plays a role in cell cycle by activating or inhibiting the Rb-p16 or p53-p21 pathway; (2) MITF also involves in DNA damage repair by engaging some signal cascades; (3) MITF can regulate multiple stress responding factors to counteract oxidative stress; and (4) MITF participates in various components of SASP production. MITF, microphthalmia-associated transcription factor; SASP, senescence-associated secretory phenotype.

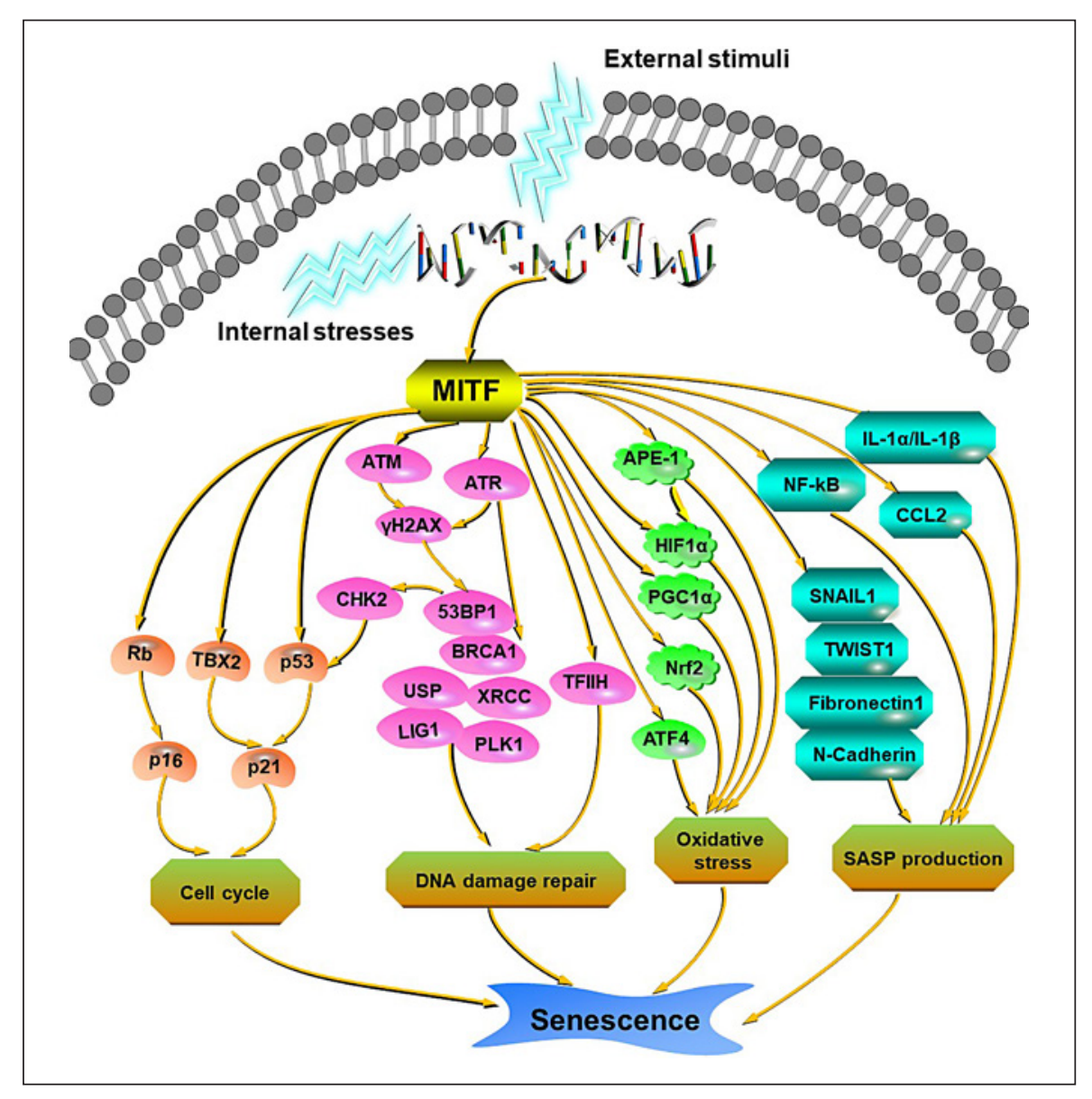

Actually, accumulated results from in vivo studies fit this notion, which shows that MITF mutant mice suffer from severe osteopetrosis. For example, the parathyroid extract injection-evoked bone resorption in MITF mutant mice was $<10 \%$ of the wild-type levels [81], and MITF mutations even caused severe osteopetrosis in mice, similar to and synergistic with the mutation of the antiapoptotic factor $\mathrm{Bcl} 2$, mechanistically via MITF-mediated upregulation of $\mathrm{Bcl} 2$ expression [82]. Moreover, studies also reported that MITF mutant mice show obvious defect in bone resorption and hormonal response [83]. In addition, human genetic studies further expound the association of MITF and bone health. Koh et al. [84] reported, based on genome sequencing of the MITF gene in 24 humansamples, that 2 polymorphisms $(+227719 \mathrm{C}>\mathrm{T}$ and $+228953 \mathrm{~A}>\mathrm{G}$ ) were significantly associated with low bone mineral density of the proximal femur in postmenopausal women. In general, MITF is closely related to the differentiation and formation of osteoclasts, and these processes affect the resorption and reconstruction of bones. Studies have tried to interfere with the activation of MITF transcription factor through various methods in an attempt to reduce the incidence of osteoporosis [85]. Hence, these results suggest that MITF can be used as a target for intervention in the treatment of osteoporosis.

\section{Cardiovascular Diseases}

As highly differentiated terminal cells, cardiomyocytes are unable to divide or proliferate. Therefore, there are only few studies focused on cardiomyocyte senescence. However, a recent study has found that aged human and mouse cardiomyocytes exhibit a classical senescence phenotype: persistent DNA damage in the telomere region, which may be driven by mitochondrial dysfunction, the classical senescence-inducing pathway, and increased SASP, which promotes myocardial hypertrophy and fibrosis. And the elimination of p16INK4a-positive cells in aged mice by genetic methods can reduce myocardial hypertrophy and fibrosis [86]. Moreover, cardiomyocytes 
have the pathological manifestation of SIPS in the cardiac disease model induced by chemotherapy drugs and obesity $[87,88]$.

MITF-H, a heart-specific isoform of MITF, is highly expressed in cardiomyocytes, making this cell type an ideal system for exploring more targets and signaling pathways than other cells that maintain a variety isoform. MITF was also reported to have differential ventricular expression in failing hearts [89]. There have been many studies on the role of the MITF in the myocardium. Specifically, the MITF-mutated mice display a cardiac hypertrophic tendency that can lead to sudden death [90]. Another study found that knockdown of MITF in cardiomyocytes can resist Ang-II-induced cardiac hypertrophic response by activating the expression of miR-541 at the transcriptional levels [91] and the cardiac hypertrophy transcription factor GATA4 [92]. Recently, a study also showed that downregulation of miR-218 can alleviate the suppression of MITF in order to improve myocardial fibrosis and stimulate angiogenesis in rats after myocardial infarction [93]. In summary, available research has suggested that MITF maintains cardiac contraction, and has a potential role in compensatory response of cardiac hypertrophy, and regulation of cardiac angiogenesis and myocardial fibrosis.

\section{Conclusion and Perspective}

Despite the well-known notion that MITF is involved in melanocyte differentiation, increasing evidence demonstrates the importance of it in aging and senescence, particularly in melanocyte senescence. MITF works for the melanocyte senescence mainly by regulating cell cycle, DNA repair, oxidative stress, and SASP production as we sketched in Figure 1. Cellular senescence is a major risk factor for many diseases and gives rise to a series of age-related pathophysiological degenerative conditions [94]. Meanwhile, senescence can also be beneficial for maintaining tissue homeostasis and preventing tumor progression [95]. As mentioned earlier, MITF is involved in the development and transformation of melanocytes, nevi, and melanoma. Therefore, it is possible to reduce the formation and metastasis of melanoma by regulating the level of MITF in order to induce melanoma cell growth arrest and promote melanoma cell senescence.

In addition to melanocytes, MITF also has a high expression level in other nonmelanocyte cells, such as RPE cells, osteoclasts, and cardiomyocytes. The different
MITF isoforms regulate unique and overlapping gene sets, demonstrating cell- and tissue-specific distribution: MITF-M is the most abundant and is widely distributed in melanocytes; MITF-A mainly exists in RPE cells; MITF-E is enriched in osteoclasts; and MITF-H is highly expressed in the heart. The abnormality of MITF may cause these cells to function improperly and result in various age-related diseases, including retinal degeneration, osteoporosis, and cardiovascular diseases, which are the leading causes of clinical adverse events and even death in the aging population. The aforementioned data demonstrate that MITF could be recognized as a player under physiological and pathogenic conditions in these cells and tissues; however, there is not enough evidence supporting the relationship between MITF and these diseases. Furthermore, MITF may participate in the oxidative stress and SASP production, which are the typical characters of senescence stated earlier. A previous study has shown that senescence can be inhibited by the regulation of a variety of senescence and stress-related proteins, thus delaying tissue aging and prolonging the life span of mice [96]. According to these results, MITF or its target gene may possess certain therapeutic potentials, which can simultaneously control the expression of multiple protective factors and ultimately benefit the treatment of degenerative diseases.

Although significant progress has been made in understanding cellular senescence with the efforts of various researchers, there are still sizable gaps in our understanding of the complex effects of MITF and senescence based on current data. For instance, the specific senescence mechanism of MITF in different cell types is still unknown. Whether the abovementioned possible mechanisms of MITF regulation of cellular senescence in melanocytes are universal or cell- or tissue-specific remains elusive. The role of other MITF isoforms in cellular senescence and other cells is also unclear. Clarifying the mechanisms of action of the transcription factor MITF and its multiple targets in senescence and discovering the temporal and spatial regulatory effects between specific cells or tissues will provide a deeper understanding of the processes associated with aging. Such clarifications may provide new insights into therapeutic interventions for aging and age-related diseases.

\section{Conflict of Interest Statement}

The authors declare that they have no conflict of interest. 


\section{Funding Sources}

This work was supported by the National Natural Science Foundation of China (Grant Nos. 81771511 and 31801013), National Clinical Research Center for Geriatrics, West China Hospital of Sichuan University (Grant No. Z2018B04), and Provincial AgencyforScience\&Technology,Sichuan(GrantNo.2016JY0125).

\section{Author Contributions}

The first draft of the manuscript was written by Jian Zhang. Hengyi Xiao and Yi Mou commented on previous versions of the manuscript. Hui Gong and Honghan Chen provided enough scientific suggestions and concrete actions during the revision. All authors read and approved the final version of the manuscript.

\section{References}

1 Hertwig P. Neue Mutationen und Kopplungsgruppen bei der Hausmaus. Z Indukt Abstammungs Vererbungsl. 1942;80:220-246.

2 Gruneberg H. Some observations on the microphthalmia gene in the mouse. J Genet. 1948;49(1):1-13

3 Marks SC, Walker DG. The hematogenous origin of osteoclasts: experimental evidence from osteopetrotic (microphthalmic) mice treated with spleen cells from beige mouse donors. Am J Anat. 1981;161(1):1-10.

4 Tachibana M, Hara Y, Vyas D, Hodgkinson C, Fex J, Grundfast K, et al. Cochlear disorder associated with melanocyte anomaly in mice with a transgenic insertional mutation. Mol Cell Neurosci. 1992;3(5):433-45.

5 Hemesath TJ, Steingrímsson E, McGill G, Hansen MJ, Vaught J, Hodgkinson CA, et al. Microphthalmia, a critical factor in melanocyte development, defines a discrete transcription factor family. Genes Dev. 1994; 8(22):2770-80.

6 Amae S, Fuse N, Yasumoto K, Sato S, Yajima I, Yamamoto $\mathrm{H}$, et al. Identification of a novel isoform of microphthalmia-associated transcription factor that is enriched in retinal pigment epithelium. Biochem Biophys Res Commun. 1998;247(3):710-5.

7 Lu SY, Li M, Lin YL. Mitf induction by RANKL is critical for osteoclastogenesis. Mol Biol Cell. 2010;21(10):1763-71.

8 Tshori S, Gilon D, Beeri R, Nechushtan H, Kaluzhny D, Pikarsky E, et al. Transcription factor MITF regulates cardiac growth and hypertrophy. J Clin Invest. 2006;116(10):267381.

9 Hayflick L, Moorhead PS. The serial cultivation of human diploid cell strains. Exp Cell Res. 1961;25:585-621.

10 Salama R, Sadaie M, Hoare M, Narita M. Cellular senescence and its effector programs. Genes Dev. 2014;28(2):99-114.

11 Kirkland JL, Tchkonia T. Clinical strategies and animal models for developing senolytic agents. Exp Gerontol. 2015;68:19-25.

12 Hodgkinson CA, Moore KJ, Nakayama A, Steingrímsson E, Copeland NG, Jenkins NA, et al. Mutations at the mouse microphthalmia locus are associated with defects in a gene encoding a novel basic-helix-loop-helix-zipper protein. Cell. 1993;74(2):395-404.

13 Tachibana M, Perez-Jurado LA, Nakayama A, Hodgkinson CA, Li X, Schneider M, et al. Cloning of MITF, the human homolog of the mouse microphthalmia gene and assignment to chromosome 3p14.1-p12.3. Hum Mol Genet. 1994;3(4):553-7.

14 Tassabehji M, Newton VE, Read AP. Waardenburg syndrome type 2 caused by mutations in the human microphthalmia (MITF) gene. Nat Genet. 1994;8(3):251-5.

15 Pogenberg V, Ogmundsdóttir MH, Bergsteinsdóttir K, Schepsky A, Phung B, Deineko $\mathrm{V}$, et al. Restricted leucine zipper dimerization and specificity of DNA recognition of the melanocyte master regulator MITF. Genes Dev. 2012;26(23):2647-58.

16 Fisher DE, Carr CS, Parent LA, Sharp PA. TFEB has DNA-binding and oligomerization properties of a unique helix-loop-helix/leucine-zipper family. Genes Dev. 1991;5(12A): 2342-52.

17 Settembre C, Di Malta C, Polito VA, Garcia Arencibia M, Vetrini F, Erdin S, et al. TFEB links autophagy to lysosomal biogenesis. Science. 2011;332(6036):1429-33.

18 Martina JA, Diab HI, Lishu L, Jeong-A L, Patange $\mathrm{S}$, Raben N, et al. The nutrient-responsive transcription factor TFE3 promotes autophagy, lysosomal biogenesis, and clearance of cellular debris. Sci Signal. 2014;7(309):ra9.

19 Zhao GQ, Zhao Q, Zhou X, Mattei MG, de Crombrugghe B. TFEC, a basic helix-loop-helix protein, forms heterodimers with TFE3 and inhibits TFE3-dependent transcription activation. Mol Cell Biol. 1993;13(8):4505-12.

20 Mahony CB, Fish RJ, Pasche C, Bertrand JY. Tfec controls the hematopoietic stem cell vascular niche during zebrafish embryogenesis. Blood. 2016;128(10):1336-45.

21 Widlund HR, Fisher DE. Microphthalamiaassociated transcription factor: a critical regulator of pigment cell development and survival. Oncogene. 2003;22(20):3035-41.

22 Lapierre LR, De Magalhaes Filho CD, McQuary PR, Chu CC, Visvikis O, Chang JT, et al. The TFEB orthologue HLH-30 regulates autophagy and modulates longevity in caenorhabditis elegans. Nat Commun. 2013;4: 2267.

23 Wang C, Niederstrasser H, Douglas PM, Lin R, Jaramillo J, Li Y, et al. Small-molecule TFEB pathway agonists that ameliorate metabolic syndrome in mice and extend C. elegans lifespan. Nat Commun. 2017;8(1):2270.

24 Steingrimsson E. All for one, one for all: alternative promoters and Mitf. Pigment Cell Melanoma Res. 2008;21(4):412-4.

25 Widlund HR, Fisher DE. Microphthalamiaassociated transcription factor: a critical regu- lator of pigment cell development and survival. Oncogene. 2003;22(20):3035-41.

26 Opdecamp K, Nakayama A, Nguyen MT, Hodgkinson CA, Pavan WJ, Arnheiter H, et al. Melanocyte development in vivo and in neural crest cell cultures: crucial dependence on the Mitf basic-helix-loop-helix-zipper transcription factor. Development. 1997;124(12):2377-86.

27 Lister JA, Robertson CP, Lepage T, Johnson SL, Raible DW. Nacre encodes a zebrafish microphthalmia-related protein that regulates neural-crest-derived pigment cell fate. Development. 1999;126(17):3757-67.

28 Bertolotto C, Abbe P, Hemesath TJ, Bille K, Fisher DE, Ortonne JP, et al. Microphthalmia gene product as a signal transducer in campinduced differentiation of melanocytes. J Cell Biol. 1998;142(3):827-35.

29 Bejar J, Hong Y, Schartl M. Mitf expression is sufficient to direct differentiation of medaka blastula derived stem cells to melanocytes. Development. 2003;130:6545-53.

30 Vachtenheim J, Borovansky J. “Transcription physiology" of pigment formation in melanocytes: central role of Mitf. Exp Dermatol. 2010;19:617-27.

31 Salti GI, Manougian T, Farolan M, Shilkaitis A, Majumdar D, Das Gupta TK, et al. Micropthalmia transcription factor: a new prognostic marker in intermediate-thickness cutaneous malignant melanoma. Cancer Res. 2000; 60(18):5012-6.

$32 \mathrm{Ko} \mathrm{H}, \mathrm{Kim}$ MM. $\mathrm{H} 2 \mathrm{O} 2$ promotes the aging process of melanogenesis through modulation of MITF and Nrf2. Mol Biol Rep. 2019; 46(2):2461-71.

33 Beausejour CM, Krtolica A, Galimi F, Narita M, Lowe SW, Yaswen P, et al. Reversal of human cellular senescence: roles of the p53 and p16 pathways. EMBO J. 2003;22:4212-22.

34 Garraway LA, Widlund HR, Rubin MA, Getz G, Berger AJ, Ramaswamy S, et al. Integrative genomic analyses identify MITF as a lineage survival oncogene amplified in malignant melanoma. Nature. 2005;436(7047):117-22.

35 Bertolotto C, Lesueur F, Giuliano S, Strub T, de Lichy M, Bille K, et al. A sumoylation-defective MITF germline mutation predisposes to melanoma and renal carcinoma. Nature. 2011;480(7375):94-8.

36 Bonet C, Luciani F, Ottavi JF, Leclerc J, Jouenne FM, Boncompagni M, et al. Deciphering the role of oncogenic MITFE318K in senescence delay and melanoma progression. J Natl Cancer Inst. 2017;109(8). 
37 Carreira S, Liu B, Goding CR. The gene encoding the T-box factor Tbx2 is a target for the microphthalmia-associated transcription factor in melanocytes. J Biol Chem. 2000; 275(29):21920-7.

38 Prince S, Carreira S, Vance KW, Abrahams A, Goding CR. Tbx2 directly represses the expression of the p21(WAF1) cyclin-dependent kinase inhibitor. Cancer Res. 2004;64(5): 1669-74.

39 Giuliano S, Cheli Y, Ohanna M, Bonet C, Beuret L, Bille K, et al. Microphthalmia-associated transcription factor controls the dna damage response and a lineage-specific senescence program in melanomas. Cancer Res. 2010;70(9):3813-22.

40 Loercher AE, Tank EM, Delston RB, Harbour JW. Mitf links differentiation with cell cycle arrest in melanocytes by transcriptional activation of INK4A. J Cell Biol. 2005;168(1):3540.

41 Carreira S, Goodall J, Aksan I, La Rocca SA, Galibert MD, Denat L, et al. Mitf cooperates with $\mathrm{Rb} 1$ and activates $\mathrm{p} 21 \mathrm{Cip} 1$ expression to regulate cell cycle progression. Nature. 2005; 433(7027):764-9.

42 Wang C, Zhao L, Su Q, Fan X, Wang Y, Gao $S$, et al. Phosphorylation of MITF by AKT affects its downstream targets and causes TP53dependent cell senescence. Int J Biochem Cell Biol. 2016;80:132-42.

43 Strub T, Giuliano S, Ye T, Bonet C, Keime C, Kobi D, et al. Essential role of microphthalmia transcription factor for DNA replication, mitosis and genomic stability in melanoma. Oncogene. 2011;30(20):2319-32.

$44 \mathrm{Ou}$ HL, Schumacher B. DNA damage responses and p53 in the aging process. Blood. 2018;131(5):488-95.

45 Laurette P, Strub T, Koludrovic D, Keime C, Le Gras S, Seberg H, et al. Transcription factor MITF and remodeller BRG1 define chromatin organisation at regulatory elements in melanoma cells. eLife. 2015;4.

46 Xia M, Chen K, Yao X, Xu Y, Yao J, Yan J, et al. Mediator MED23 links pigmentation and DNA repair through the transcription factor MITF. Cell Rep. 2017;20(8):1794-804

47 Seoane M, Buhs S, Iglesias P, Strauss J, Puller AC, Müller J, et al. Lineage-specific control of TFIIH by MITF determines transcriptional homeostasis and DNA repair. Oncogene. 2019;38(19):3616-35.

48 Podda M, Traber MG, Weber C, Yan LJ, Packer L. UV-irradiation depletes antioxidants and causes oxidative damage in a model of human skin. Free Radic Biol Med. 1998;24(1): 55-65.

49 Jiménez-Cervantes C, Martinez-Esparza M, Perez C, Daum N, Solano F, García-Borrón JC. Inhibition of melanogenesis in response to oxidative stress: transient downregulation of melanocyte differentiation markers and possible involvement of microphthalmia transcription factor. J Cell Sci. 2001;114:2335-44.

50 Liu F, Fu Y, Meyskens FL. MiTF regulates cellular response to reactive oxygen species through transcriptional regulation of APE-1/ REF-1. J Invest Dermatol. 2009;129(2):42231.

51 Busca R, Berra E, Gaggioli C, Khaled M, Bille K, Marchetti B, et al. Hypoxia-inducible factor 1 alpha\} is a new target of microphthalmia-associated transcription factor (MITF) in melanoma cells. J Cell Biol. 2005;170:49-59.

52 Vazquez F, Lim JH, Chim H, Bhalla K, Girnun G, Pierce K, et al. PGC1 a expression defines a subset of human melanoma tumors with increased mitochondrial capacity and resistance to oxidative stress. Cancer Cell. 2013; 23(3):287-301.

53 Haq R, Shoag J, Andreu-Perez P, Yokoyama S, Edelman H, Rowe GC, et al. Oncogenic BRAF regulates oxidative metabolism via PGC1 $\alpha$ and MITF. Cancer Cell. 2013;23(3): 302-15.

54 Jackson E, Heidl M, Imfeld D, Meeus L, Schuetz R, Campiche R, et al. Discovery of a highly selective MC1R agonists pentapeptide to be used as a skin pigmentation enhancer and with potential anti-aging properties. Int J Mol Sci. 2019;20(24):6143.

55 Jiang W, Li S, Chen X, Zhang W, Chang Y, He $\mathrm{Y}$, et al. Berberine protects immortalized line of human melanocytes from $\mathrm{H} 2 \mathrm{O} 2$-induced oxidative stress via activation of $\mathrm{Nrf} 2$ and Mitf signaling pathway. J Dermatol Sci. 2019; 94(1):236-43

56 Hseu YC, Ho YG, Mathew DC, Yen HR, Chen $\mathrm{XZ}$, Yang HL, et al. The in vitro and in vivo depigmenting activity of Coenzyme Q10 through the down-regulation of $\alpha-\mathrm{MSH}$ signaling pathways and induction of Nrf2/AREmediated antioxidant genes in UVA-irradiated skin keratinocytes. Biochem Pharmacol. 2019;164:299-310

57 Falletta P, Sanchez-Del-Campo L, Chauhan J, Effern M, Kenyon A, Kershaw CJ, et al. Translation reprogramming is an evolutionarily conserved driver of phenotypic plasticity and therapeutic resistance in melanoma. Genes Dev. 2017;31(1):18-33.

58 Coppe JP, Patil CK, Rodier F, Sun Y, Munoz DP, Goldstein J, et al. Senescence-associated secretory phenotypes reveal cell-nonautonomous functions of oncogenic ras and the p53 tumor suppressor. PLoS Biol. 2008;6:285368.

59 Sun Y, Coppé JP, Lam EW. Cellular senescence: the sought or the unwanted? Trends Mol Med. 2018;24(10):871-85.

60 Kholmanskikh O, van Baren N, Brasseur F, Ottaviani S, Vanacker J, Arts N, et al. Interleukins 1alpha and 1beta secreted by some melanoma cell lines strongly reduce expression of MITF-M and melanocyte differentiation antigens. Int J Cancer. 2010;127(7):1625-36.

61 Ohanna M, Giuliano S, Bonet C, Imbert V, Hofman V, Zangari J, et al. Senescent cells develop a PARP-1 and nuclear factor-\{kappa\} b-associated secretome (PNAS). Genes Dev. 2011;25(12):1245-61.

62 Ohanna M, Cheli Y, Bonet C, Bonazzi VF, Allegra M, Giuliano S, et al. Secretome from senescent melanoma engages the STAT3 pathway to favor reprogramming of naive melanoma towards a tumor-initiating cell phenotype. Oncotarget. 2013;4(12):2212-24.

63 Song C, Piva M, Sun L, Hong A, Moriceau G, Kong X, et al. Recurrent tumor cell-intrinsic and -extrinsic alterations during mapki-induced melanoma regression and early adaptation. Cancer Discov. 2017;7(11):1248-65.

64 Voigt AP, Mulfaul K, Mullin NK, FlammeWiese MJ, Giacalone JC, Stone EM, et al. Single-cell transcriptomics of the human retinal pigment epithelium and choroid in health and macular degeneration. Proc Natl Acad Sci U S A. 2019;116(48):24100-7.

65 Amae S, Fuse N, Yasumoto K, Sato S, Yajima I, Yamamoto H, et al. Identification of a novel isoform of microphthalmia-associated transcription factor that is enriched in retinal pigment epithelium. Biochem Biophys Res Commun. 1998;247(3):710-5

66 Ma X, Hua J, Zheng G, Li F, Rao C, Li H, et al. Regulation of cell proliferation in the retinal pigment epithelium: differential regulation of the death-associated protein like-1 DAPL1 by alternative MITF splice forms. Pigment Cell Melanoma Res. 2018;31(3):411-22.

67 Moller A, Eysteinsson T, Steingrimsson E Electroretinographic assessment of retinal function in microphthalmia mutant mice. Exp Eye Res. 2004;78:837-48

68 Ou J, Bharti K, Nodari A, Bertuzzi S, Arnheiter $\mathrm{H}$. Vax $1 / 2$ genes counteract Mitf-induced respecification of the retinal pigment epithelium. PLoS One. 2013;8(3):e59247.

69 Wen B, Li S, Li H, Chen Y, Ma X, Wang J, et al. Microphthalmia-associated transcription factor regulates the visual cycle genes RLBP1 and RDH5 in the retinal pigment epithelium. Sci Rep. 2016;6:21208.

70 Hua J, Chen H, Chen Y, Zheng G, Li F, Qu J, et al. Mitf acts as an anti-oxidant transcription factor to regulate mitochondrial biogenesis and redox signaling in retinal pigment epithelial cells. Exp Eye Res. 2018;170:138-47.

71 Han S, Chen J, Hua J, Hu X, Jian S, Zheng G, et al. Mitf protects against oxidative damageinduced retinal degeneration by regulating the Nrf2 pathway in the retinal pigment epithelium. Redox Biol. 2020;34:101537.

72 Kaarniranta K, Uusitalo H, Blasiak J, Felszeghy S, Kannan R, Kauppinen A, et al. Mechanisms of mitochondrial dysfunction and their impact on age-related macular degeneration. Prog Retin Eye Res. 2020;79:100858.

73 Sankar U, Patel K, Rosol TJ, Ostrowski MC. RANKL coordinates cell cycle withdrawal and differentiation in osteoclasts through the cyclindependent kinase inhibitors p27kip1 and p21cip1. J Bone Miner Res. 2004;19(8):1339-48.

74 Marks SJ. Pathogenesis of osteopetrosis in the microphthalmic mouse: reduced bone resorption. Am J Anat. 1977;149(2):269-75.

75 Asai K, Funaba M, Murakami M. Enhancement of RANKL-induced MITF-E expression and osteoclastogenesis by TGF- $\beta$. Cell Biochem Funct. 2014;32(5):401-9. 
76 Ishii J, Kitazawa R, Mori K, McHugh KP, Morii E, Kondo T, et al. Lipopolysaccharide suppresses RANK gene expression in macrophages by down-regulating PU.1 and MITF. J Cell Biochem. 2008;105(3):896-904.

77 Lu SY, Li M, Lin YL. Mitf regulates osteoclastogenesis by modulating NFATC1 activity. Exp Cell Res. 2014;328(1):32-43.

78 Pang M, Rodríguez-Gonzalez M, Hernandez M, Recinos CC, Seldeen KL, Troen BR, et al. AP-1 and Mitf interact with NFATC1 to stimulate cathepsin $\mathrm{k}$ promoter activity in osteoclast precursors. J Cell Biochem. 2019;120(8): 12382-92.

79 Thesingh CW, Scherft JP. Fusion disability of embryonic osteoclast precursor cells and macrophages in the microphthalmic osteopetrotic mouse. Bone. 1985;6(1):43-52.

80 McGill GG, Horstmann M, Widlund HR, Du J, Motyckova G, Nishimura EK, et al. $\mathrm{Bcl} 2$ regulation by the melanocyte master regulator Mitf modulates lineage survival and melanoma cell viability. Cell. 2002; 109(6):707-18.

81 Marks SJ. Pathogenesis of osteopetrosis in the microphthalmic mouse: reduced bone resorption. Am J Anat. 1977;149(2):26975.

82 McGill GG, Horstmann M, Widlund HR, Du J, Motyckova G, Nishimura EK, et al. Bcl2 regulation by the melanocyte master regulator Mitf modulates lineage survival and melanoma cell viability. Cell. 2002;109(6):707-18.
83 Raisz LG, Simmons HA, Gworek SC, Eilon G. Studies on congenital osteopetrosis in microphthalmic mice using organ cultures: impairment of bone resorption in response to physiologic stimulators. J Exp Med. 1977; 145(4):857-65.

84 Koh JM, Kim GS, Oh B, Lee JY, Park BL, Shin $\mathrm{HD}$, et al. Microphthalmia-associated transcription factor polymorphisms and association with bone mineral density of the proximal femur in postmenopausal women. Mol Cells. 2007;23(2):246-51.

85 Courtial N, Smink JJ, Kuvardina ON, Leutz A, Göthert JR, Lausen J, et al. Tal1 regulates osteoclast differentiation through suppression of the master regulator of cell fusion DCSTAMP. FASEB J. 2012;26(2):523-32.

86 Anderson R, Lagnado A, Maggiorani D, Walaszczyk A, Dookun E, Chapman J, et al. Length-independent telomere damage drives post-mitotic cardiomyocyte senescence. EMBO J. 2019;38(5):e100492.

87 Liao CH, Hsiao YM, Lin CH, Yeh CS, Wang JC, $\mathrm{Ni} \mathrm{CH}$, et al. Induction of premature senescence in human lung cancer by fungal immunomodulatory protein from ganoderma tsugae. Food Chem Toxicol. 2008;46(5): 1851-9.

88 Niemann B, Chen Y, Teschner M, Li L, Silber RE, Rohrbach S, et al. Obesity induces signs of premature cardiac aging in younger patients: the role of mitochondria. J Am Coll Cardiol. 2011;57(5):577-85.
89 Gao Z, Xu H, DiSilvestre D, Halperin VL, Tunin R, Tian Y, et al. Transcriptomic profiling of the canine tachycardia-induced heart failure model: global comparison to human and murine heart failure. J Mol Cell Cardiol. 2006;40(1):76-86.

90 Tshori S, Sonnenblick A, Yannay-Cohen N, Kay G, Nechushtan H, Razin E, et al. Microphthalmia transcription factor isoforms in mast cells and the heart. Mol Cell Biol. 2007; 27(11):3911-9.

91 Liu F, Li N, Long B, Fan YY, Liu CY, Zhou QY, et al. Cardiac hypertrophy is negatively regulated by miR-541. Cell Death Dis. 2014;5: e1171.

92 Mehta G, Kumarasamy S, Wu J, Walsh A, Liu $\mathrm{L}$, Williams $\mathrm{K}$, et al. MITF interacts with the SWI/SNF subunit, BRG1, to promote GATA4 expression in cardiac hypertrophy. J Mol Cell Cardiol. 2015;88:101-10.

93 Qian L, Pan S, Shi L, Zhou Y, Sun L, Wan Z, et al. Downregulation of microRNA-218 is cardioprotective against cardiac fibrosis and cardiac function impairment in myocardial infarction by binding to MITF. Aging. 2019; 11(15):5368-88.

94 Dong X, Milholland B, Vijg J. Evidence for a limit to human lifespan. Nature. 2016; 538(7624):257-9.

95 Hartl FU. Cellular homeostasis and aging. Annu Rev Biochem. 2016;85:1-4.

96 Du WW, Yang W, Fang L, Xuan J, Li H, Khorshidi A, et al. mir-17 extends mouse lifespan by inhibiting senescence signaling mediated by MKP7. Cell Death Dis. 2014;5:e1355. 